\title{
Armazenamento de Curto Prazo e Velocidade de Processamento Explicam a Relação entre Memória de Trabalho e o Fator $g$ de Inteligência
}

\author{
Roberto Colom \\ Universidad Autónoma de Madrid \\ Carmen E. Flores-Mendoza ${ }^{1}$ \\ Universidade Federal de Minas Gerais
}

\begin{abstract}
RESUMO - O presente artigo constitui a apresentação e discussão das evidências empíricas derivadas da análise das relações entre dois construtos-chaves do processamento humano de informação e da psicologia das diferenças individuais, a saber: a memória de trabalho e o fator geral de inteligência $(g)$. Os resultados publicados avaliam a presença de um isomorfismo entre ambos construtos, embora ainda se desconheça a resposta à pergunta sobre o porquê desse isomorfismo. Enquanto algumas propostas favorecem uma explicação baseada em construtos tais como o controle da atenção, outras se inclinam pelo papel da capacidade para armazenar transitoriamente a informação e, em menor grau, da velocidade de processamento. Descrevem-se as principais discussões acadêmicas e se apresentam os resultados obtidos em recentes investigações conduzidas pelos autores. Conclui-se que os componentes de armazenamento e velocidade de processamento podem constituir a fonte de explicação da relação entre o construto de memória de trabalho e o fator $g$.
\end{abstract}

Palavras-chave: memória de trabalho; memória de curto prazo; velocidade de processamento; fator $g$.

\section{Short-term Storage and Processing Speed Explain the Relationship between Working Memory and the Intelligence $g$ Factor}

\begin{abstract}
The present article presents and discusses the empirical evidences derived from the analysis of the relationship between two key constructs of both human information processing and psychology of individual differences, namely: working memory and general factor of intelligence $(g)$. Empirical evidences supports the isomorphism between both constructs although the reason why such isomorphism is kept remains unknown. In some studies results support the important role of controlled attention, while others recognize the favour short-term storage (and short-term recognition speed) as nuclear components underlying such relationship. The core academic discussions around this topic, as well as data collected in recent investigations conducted by the authors are described. The main findings of our group are consistent with the perspective that the capacity for short-term storage, as well as for short-term recognition speed, account for the strong relationship between the working memory and the $g$ factor.
\end{abstract}

Key words: working memory; short term memory; processing speed; $g$ factor.

Em um artigo passado (2001) apresentamos os fundamentos teóricos da memória de trabalho (MT) e do fator $g$. Dizíamos que o primeiro representava um sistema de memória em que os sujeitos armazenavam transitoriamente a informação e a submetiam a um trabalho de processamento, diferentemente da memória de curto prazo (MCP) em que os sujeitos apenas armazenavam a informação em um breve período de tempo. Do segundo construto, o fator $g$, dizíamos que este podia ser concebido como uma fonte comum de diferenças individuais em todos os testes mentais até então empregados e que estaria associado, em última instância, a diversos fenômenos comportamentais e sociais como rendimento ocupacional, saúde, liderança, interesses, renda econômica ou resposta à psicoterapia. Por último, afirmávamos que ambos construtos, a MT e o fator $g$, compartilham

1 Endereço: Av. Antônio Carlos, 6627, FAFICH, Depto. de Psicologia, Gab. 4042, Laboratório de Avaliação das Diferenças Individuais, Universidade Federal de Minas Gerais, Belo Horizonte, MG, Brasil 31270-901. E-mail: carmencita@fafich.ufmg.br recursos mentais relacionados à capacidade e à velocidade de processamento de informação.

Atualmente existe um razoável cabedal de informações, produto de diversos empreendimentos investigativos, sobre a relação entre MT e inteligência $(g)$ e sobre a estrutura e funcionamento da MT. Como se verá ao longo do presente artigo, os resultados dessas e das nossas próprias investigações, largamente vêm mostrando que a hipótese teórica, de fato, era pertinente: a capacidade de armazenamento e a velocidade de processamento resultam necessários e suficientes para explicar a relação entre MT e $g$.

\section{O debate sobre MT, MCP e $g$}

Desde os anos 1960 (época em que aparece pela primeira vez a expressão “memória de trabalho"), diversos trabalhos mostraram evidências de uma possível relação entre esse construto e as habilidades cognitivas (Richardson, 1996). No entanto, foi o trabalho de Carpenter, Just e Shell (1990) o ponto de partida para um despertar científico sobre a importância da MT na cognição humana. Em síntese, nesse trabalho 
afirmou-se que o desempenho em testes de inteligência como as Matrizes Progressivas de Raven requer a manutenção de determinado número de regras subjacentes aos itens. Um maior número de regras produz necessariamente uma maior complexidade do item. Nessa situação, o êxito em resolver o item depende da capacidade do sujeito em manter ativas as regras que esse item demanda. E essa capacidade era a memória de trabalho. Os resultados obtidos pelos pesquisadores pareceram confirmar tal suposição. Os sujeitos com maior capacidade de memória de trabalho resolveram maior número de itens e, portanto, resolveram itens mais complexos do que sujeitos com menor capacidade de memória de trabalho (Colom \& Flores-Mendoza, 2006).

Kyllonen e Christal (1990), em um árduo e impressionante trabalho, avaliaram 2000 sujeitos com uma bateria de tarefas que incluía provas de raciocínio, de memória de trabalho, de velocidade e de conhecimento geral. Os resultados indicaram altas correlações entre memória de trabalho e raciocínio $(0,80$ a 0,88$)$. Tais resultados mostravam, portanto, que o êxito nas tarefas cognitivas dependia da habilidade em manter ativa a informação e da capacidade em processá-la. Posteriormente, Kyllonen (1996) postulou que a MT seria o próprio fator $g$ de Spearman.

Engle, Tuholski, Laughlin e Conway (1999) analisaram três medidas verbais de MCP, três medidas verbais de MT e os testes de inteligência Cattell's Culture Fair Test e as Matrizes Progressivas de Raven, tidos como medidas de inteligência fluída ou Gf (capacidade cognitiva relacionada à maturação do córtex, fatores genéticos e funcionamento neurológico). Suas análises revelaram uma alta relação entre MCP e MT, mas unicamente o componente específico de MT (aquele não vinculado ao armazenamento transitório) se relacionava significativamente às medidas de Gf. No caso do fator latente de inteligência cristalizada ou Gc (capacidade executiva, produto da experiência e da prática, que acompanha o repertório cognitivo) extraído de medidas verbais e numéricas, as diferenças individuais foram preditas tanto pelo componente de armazenamento (MCP) como pelo componente específico de MT (não relacionado ao armazenamento transitório).

Conway, Cowan, Bunting, Therriault e Minkoff (2002) avaliaram 120 estudantes universitários com três tarefas de MT, quatro medidas de MCP, três medidas de velocidade de processamento, e com medidas de Gf (Matrizes Progressivas de Raven e o Cattell's Culture Fair Test). Usando um modelo alternativo ao empregado por Engle e cols. (1999), os pesquisadores chegaram a resultados similares, isto é, encontraram uma alta correlação entre MCP e MT. Encontraram também que o componente específico de MT, não relacionado ao armazenamento transitório, predizia as diferenças individuais em $g$.

Kane e cols. (2004) empregaram diversas medidas de MT e MCP de ordem verbal e viso-espacial assim como diversas medidas de habilidade cognitiva. Esses autores, apesar de pertencerem à equipe de Engle e de Conway, empregaram um terceiro tipo de modelo confirmatório para chegar à conclusão, menos sólida, de que o componente de MT independente do armazenamento predizia as diferenças individuais em Gf. No entanto, também encontraram que um fator latente correspondente ao armazenamento espacial predizia Gf com igual intensidade. O estudo empírico de Kane e cols. (2004) tem sido fortemente questionado por nossa equipe (ver detalhes em Colom, Abad, Rebollo \& Shih, 2005).

Ackerman, Beier e Boyle (2005) conduziram uma metaanálise examinando a relação entre MT e $g$, assim como também entre MCP e $g$. O estudo esteve baseado em estudos publicados na literatura entre 1872 e 2002. Classificaram os testes de habilidade cognitiva em diferentes categorias em que se incluíam habilidades de ordem verbal, quantitativa, espacial e raciocínio geral. As medidas de MT também foram classificadas pelo conteúdo e se elas tinham ou não incluído processamento simultâneo de diferentes conteúdos. O principal resultado dessa meta-análise é que a correlação entre MCP e $g$ resultou equivalente à correlação entre MT e $g$.

Considerando esses e outros estudos (Ackerman, Beier \& Boyle, 2002; Stauffer, Ree \& Carretta, 1996; Süß, Oberauer, Wittman, Wilhelm \& Shulze, 2002) parecia haver pouca dúvida em considerar que a MT e $g$ compartilham a mesma fonte de variância, o que reforçaria a visão de MT como um sistema unitário. Contudo, os estudos de Shah e Miyake (1996) mostraram evidências de uma separação dos recursos de MT. Esses autores indicaram que a MT verbal seria independente da MT espacial (embora suas correlações não sejam tão expressivas dessa conclusão).

Seguindo essa linha de raciocínio, Mackintosh e Bennett (2003) examinaram medidas verbais, espaciais e quantitativas assim como testes de inteligência fluída, inteligência cristalizada e habilidade espacial por meio de compostos (uma medida de MT e uma medida cognitiva) para o fator latente Gf (fator latente fluído), para Gc (fator latente cristalizado) e para Gv (fator latente viso-espacial). Utilizando análises confirmatórias, Mackintosh e Bennett (2003) encontraram altas correlações entre Gc e Gf $(0,67)$ e entre Gf e Gv $(0,73)$. A correlação, no entanto, diminuiu para Gc e Gv, evidenciando dessa forma uma nítida separação entre o domínio verbal e o domínio espacial. Os autores concluíram que MT podia ser fracionado em diversos componentes.

Por outro lado, advogou-se que MT seria indistinguível de MCP, uma vez que a MCP representaria unicamente armazenamento e MT representaria armazenamento + processamento de informação. Essa superposição dos componentes de MT e MCP levaria a posições acadêmicas díspares. Assim, por exemplo, Anderson (1990) sugeriu que MCP e MT são construtos similares; Seamon e Kenrick (1994) postularam que MT é um subconjunto de MCP, e Cowan (1995) propôs que MCP é um subconjunto de MT. Várias investigações foram efetuadas, ao nível da variável latente, para elucidar essa questão.

Engle e cols. (1999) encontraram uma correlação de 0,68 entre os fatores latentes de MT e MCP (utilizando medidas verbais); Conway e cols. (2002) encontraram uma correlação de 0,82 (utilizando medidas verbais); Miyake, Friedman, Rettinger, Shah, e Hegarty (2001) encontraram um índice de 0,86 (utilizando medidas espaciais). Esses resultados revelaram, portanto, uma consistente associação entre MT e MCP. No entanto, deve-se registrar que esses estudos consideraram unicamente ou tarefas verbais ou tarefas espaciais. A única investigação em que se empregaram tarefas diversas foi a de Kane e cols. (2004). Eles não informaram a correlação entre MCP e MT, mas nossa equipe, trabalhando com a matriz de dados cedida pelos autores, calculou a 
correlação em 0,99 (Colom, Abad, Rebollo \& Shih, 2005). Esse resultado, portanto, reforça a posição de haver extrema dificuldade em distinguir MCP e MT, ao nível de construto. Maiores investigações utilizando tarefas que representem diversidade de domínios se fazem necessárias. Uma outra questão a ser elucidada é sobre a relação existente entre a MCP e $g$ e entre a MT e $g$.

A meta-análise de Ackerman e cols. (2005) aponta que a correlação bruta entre MCP e $g$ é de 0,28 enquanto que a correlação entre MT e $g$ é 0,36. Também, o estudo de Mukunda e Hall (1992), que incluía estudos publicados entre 1976 e 1989, apontou uma correlação de 0,26 entre dados brutos de medidas de MCP e medidas de inteligência, e uma relação entre MT e inteligência de 0,28. Esses resultados indicavam que MCP e MT eram igualmente preditores de $g$. No entanto, recorde-se que Engle e cols. (1999), Conway e cols. (2002) e Kane e cols. (2004) encontraram que MT, mas não MCP, predizia as diferenças individuais em inteligência. Não há, portanto, uma confirmação robusta sobre a predição de MCP e MT em relação a $g$.

Nesse sentido, três questões permanecem abertas no campo de investigação da MT e capacidade cognitiva, a saber: Qual é a natureza de MT (se unitário ou de domínio específico)? Qual a relação entre MCP e MT? Qual é o melhor preditor de $g$ (se MCP ou MT)? Essas três questões são as que nos instigaram a realizar diversos estudos utilizando medidas de MCP, MT e PS, algumas adaptadas da literatura e outras construídas por nossa equipe ${ }^{2}$. Para tanto, utilizamos as seguintes técnicas de análise quantitativas:

- Análise de Regressão Hierárquica: permite averiguar a relação entre uma série de variáveis preditoras e uma medida critério, levando em consideração a co-variação entre as variáveis preditoras. Portanto, essa técnica permite averiguar a capacidade de predição genuína das variáveis preditoras.

- Análise Hierárquica Schmid-Leiman. Trata-se de uma técnica de análise fatorial em que os fatores de ordem superior explicam o máximo que puderem as correlações existentes entre as variáveis; enquanto que os fatores de ordem inferior são reduzidos a fatores residuais não correlacionados entre eles e com os fatores de ordem superior (Carroll, 1993; Jensen \& Weng, 1994).

- Análise Fatorial Confirmatória (ou CFA). Trata-se de uma técnica para testar ou confirmar um relacionamento de variáveis preestabelecido pelo pesquisador. Seguindo a recomendação de Browne e Cudeck (1992), decidimos aceitar modelos de CFAs quando o índice de adequação RMSEA (Root-Mean Square Error of Aproximation) apresentasse valor menor que 0,08 . Também, considerouse a razão CMIN/df, cujos valores estejam próximos de 2,0 .

- Structural Equation Modelling (ou SEM). Trata-se de uma técnica em que se analisa simultaneamente uma série de relacionamentos entre as variáveis dependentes. A técnica combina aspectos de regressão múltipla e de análise fatorial. A técnica é conhecida também como abordagem de variável latente. Tanto as análises SEM

2 Ver descrição de tarefas MCP, MT e PS no Anexo 1. quanto os CFAs foram calculados mediante os programas AMOS ou LISREL. Para maiores detalhes sobre o comportamento psicométrico das variáveis, assim como sobre os métodos de estimação, o leitor deve se remeter aos artigos originais.

Essas técnicas subsidiaram a interpretação dos resultados de nossas investigações e permitiram responder às três questões que a seguir se apresentam.

\section{Qual é a natureza de MT (unitária ou específica)?}

Os primeiros estudos que efetuamos sobre MT e diferenças individuais em inteligência contaram com a participação de 594 sujeitos adultos aos quais aplicamos tarefas de MT e medidas de velocidade de processamento (Colom, Rebollo, Palacios, Juan-Espinosa \& Kyllonen, 2004). Nesses estudos empregamos, também, testes de Gc, Gv, Gf e PS (velocidade processamento). O objetivo era o de estabelecer o grau de relação entre MT e $g$. Para tanto, submetemos os dados à análise confirmatória (CFA). Nos três estudos independentes demonstramos que o fator latente que melhor predizia $g$ correspondia a MT. O valor resultou tão elevado $(0,96)$ que se chegou à conclusão de que $g$ e MT podiam ser considerados como construtos isomórficos.

Considerando os resultados do estudo de Mackintosh e Bennett (2003), em que obtiveram fatores específicos de MT, decidimos realizar um estudo de réplica incrementando o tamanho amostral e o número de medidas que permitiam definir os fatores latentes de interesse (Colom \& Shih, 2004). Neste, submetemos 226 sujeitos universitários a um total de 12 provas, sendo seis tarefas MT (duas verbais, duas numéricas e duas espaciais) e seis provas de habilidade psicométrica (no estudo de Mackintosh e Bennett utilizaram-se três tarefas de MT e três de habilidades cognitivas em 138 sujeitos).

Em nosso estudo, as provas cognitivas avaliavam Gc, Gf e Gv. Seguindo o método utilizado por Mackintosh e Bennett (2003), dessas 12 provas, obtivemos seis fatores latentes primários e, depois, três fatores de segunda ordem: Gc (definido pelo MT verbal e habilidade verbal); Gf (definido pelo MT quantitativo e inteligência fluída) e Gv (definido pelo MT espacial e habilidade espacial). O modelo hierárquico de fatores latentes mostrou-se adequado aos dados $\left[\chi^{2}(45)=92,02 ; \mathrm{CMIN} / d f=2,04 ; \mathrm{RMSEA}=0,068\right]$. Diferentemente de Mackintosh e Bennett (2003), as correlações que obtivemos entre Gc, Gf e Gv foram maiores do que 0,90 (Figura 1). As altas correlações entre os fatores de segunda ordem nos sugeriram a presença de um único fator latente. Assim, submetemos os três fatores de segunda ordem a uma CFA para testar um modelo restrito. Os resultados mostraram que o modelo restrito de um fator adequava-se aos dados $\left[\chi^{2}(48)=93,67 ; \mathrm{CMIN} / d f=1,95\right.$; RMSEA $=0,065]$.

Os resultados do modelo hierárquico e do modelo restrito evidenciaram, portanto, a natureza unitária do sistema de MT, rejeitando o fracionamento de MT em diferentes componentes de inteligência como tinham proposto Mackintosh e Bennett (2003). Uma evidência adicional consistente com a visão unitária é que a correlação entre o fator latente correspondente a MT e o fator latente correspondente a $g$ resultou em um valor de 0,86 (Colom \& Shih, 2004). 


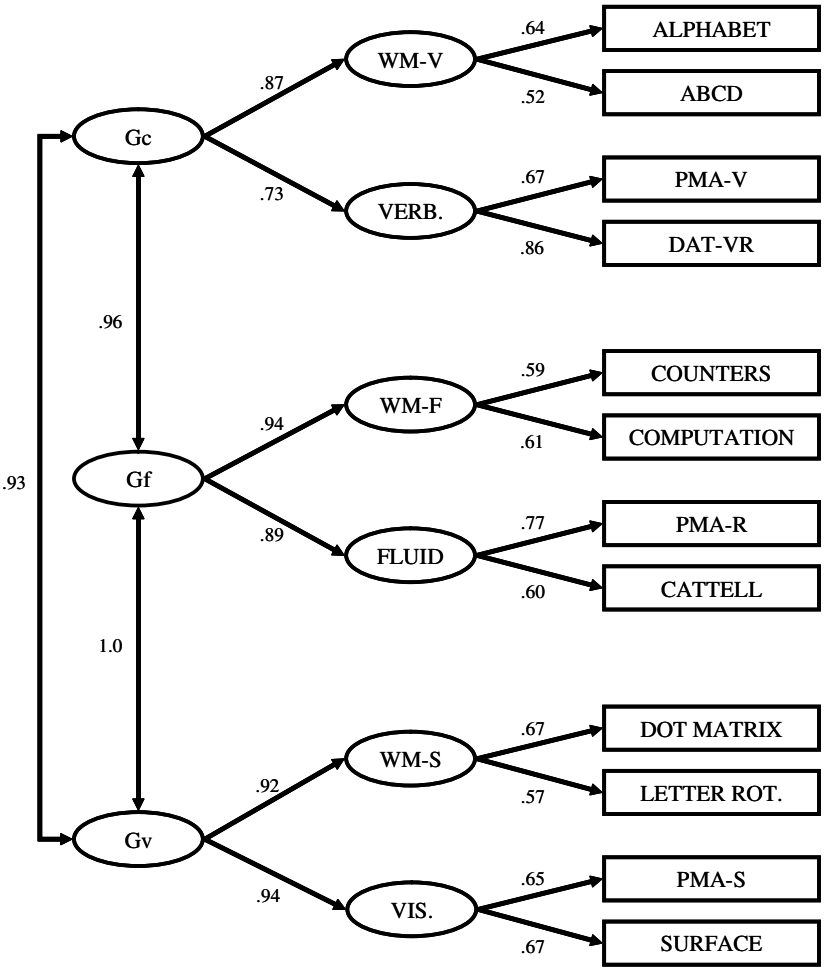

Figura 1. Modelo hierárquico para Gv, Gf e Gv.

Nota da Figura 1. Gc = inteligência cristalizada, Gf = inteligência fluida, $\mathrm{Gv}=$ inteligência espacial, $\mathrm{WM}-\mathrm{V}=$ memória de trabalho verbal, VERB . = inteligência verbal, $\mathrm{WM}-\mathrm{F}=$ memória de trabalho numérica/fluida, FLUID = inteligência fluida, WM-S = memória de trabalho espacial, VIS. $=$ inteligência espacial. ALPHABET $=$ tarefa de alfabeto, $\mathrm{ABCD}=$ tarefa de ABCD, PMA-V = subteste de vocabulário do Primary Mental Abilities Battery, DAT-VR = subteste de raciocínio verbal da Differential Aptitude Test Battery, COUNTERS $=$ tarefa de contadores, COMPUTATION $=$ tarefa de amplitude de cálculo, PMA-R = subteste de raciocínio indutivo do Primary Mental Abilities Battery, CATTELL = subtestes 1 e 3 do teste livre de influências culturais de R.B. Cattell, DOT MATRIX = tarefa de verificação de matrizes e memorização de pontos, LETTER ROT. $=$ tarefa de rotação de letras e memorização de posições, PMA-S = subteste de rotação mental do Primary Mental Abilities Battery, SURFACE = teste de desenvolvimento de superfícies.

Todos os coeficientes de regressão estão padronizados. Os valores residuais foram omitidos visando uma maior clareza dos resultados. E eles podem ser facilmente calculados: eleva-se ao quadrado os valores dos coeficientes de regressão que alcançam uma determinada variável endógena ou exógena e subtrai-se de 1 . Por exemplo: $0,87^{2}=0,76, \operatorname{logo} 1-0,76=0,24$ constituiria o residual de WM-V. No caso de haver mais de um valor, deve-se somar o resultado e subtrair de 1 .

\section{Qual é a "real" associação entre MCP e MT?}

Como dito anteriormente, a MCP teoricamente representa armazenamento transitório de informação e MT representa armazenamento + processamento transitório de informação. Parece obvio supor, então, que ambos os construtos compartilhem, em algum grau, as mesmas fontes de variância. Para verificar tal questão decidimos analisar fatorialmente essa suposta relação a partir do desempenho de 403 sujeitos universitários em 12 medidas de memória, sendo seis MCP e seis MT (Colom, Shih, Flores-Mendoza \& Quiroga, no prelo). Os escores brutos dessas medidas foram padronizados para obter um composto verbal, um quantitativo e um outro espacial tanto para MCP quanto para MT. Logo, a partir das médias desses compostos definimos escores gerais para MCP e para MT. A correlação bruta desses escores gerais foi de $0,70(p<0,01)$. Finalmente, submetemos a matriz de correlações entre os compostos específicos a uma análise fatorial exploratória, especificamente a principal axis factoring (PAF). Essa análise revelou um único e forte fator com eigenvalue de 3,14 que explicava 43\% da variância (Tabela 1 ). Tal resultado indicava, portanto, que todos os componentes de MCP e MT não eram claramente distinguíveis.

Em um estudo transcultural (Colom, Flores-Mendoza \& Rebollo, 2003) testamos 187 sujeitos, sendo 71 brasileiros e 116 espanhóis, com idade entre 14 e 47 anos de idade. Na amostra brasileira a medida de inteligência foi as Matrizes Progressivas de Raven, enquanto que na amostra espanhola a medida cognitiva foi o Primary Mental Abilities Test. A ambas amostras aplicou-se uma bateria informatizada de oito tarefas de memória. Quatro delas exigiam apenas armazenamento e evocação transitória de informação, representando a memória de curto prazo (ou MCP) e as outras quatro exigiam armazenamento + processamento transitório de informação, representando a memória de trabalho (ou MT). A matriz de correlações mostrou altos coeficientes de associação entre as tarefas de MT e MCP (Tabela 2). Logo, submetemos essa matriz de correlações a uma análise fatorial na qual extraímos um único fator que explicava quase $70 \%$ da variância comum. Finalmente, correlacionamos os escores fatoriais com os escores obtidos nos testes de inteligência. Na amostra brasileira obtivemos uma correlação de Pearson de 0,69 e na amostra espanhola obtivemos uma correlação de 0,71 . Esses resultados evidenciaram, portanto, a existência de um recurso geral cognitivo que representa MT e que está associado a uma medida de inteligência.

Tabela 1. Matriz de Correlações para os escores de compostos de MCP e MT e matriz fatorial.

\begin{tabular}{|c|c|c|c|c|c|c|c|c|}
\hline & MCP-V & MCP-Q & MCP-S & MT-V & MT-Q & MT-S & Fator & $\mathbf{h}^{2}$ \\
\hline MCP-V & & .70 & .37 & .48 & .43 & .33 & .74 & .54 \\
\hline MCP-Q & & & .34 & .46 & .48 & .36 & .75 & .56 \\
\hline MCP-S & & & & .29 & .45 & .45 & .56 & .31 \\
\hline MT-V & & & & & .40 & .38 & .61 & .38 \\
\hline MT-Q & & & & & & .47 & .68 & .47 \\
\hline MT-S & & & & & & & .58 & .34 \\
\hline \% Variância & & & & & & & & 43 \\
\hline
\end{tabular}

Nota. os compostos de MCP e MT são de tarefas de conteúdo verbal (V), quantitativo (Q) e espacial (S). 
Memória, Velocidade de Processamento e Inteligência.

Tabela 2. Estatística descritiva, matriz de correlações (a matriz residual é apresentada abaixo da diagonal) e matriz fatorial.

\begin{tabular}{|c|c|c|c|c|c|c|c|c|c|c|c|}
\hline \multirow{2}{*}{ Tarefas e Medidas } & \multicolumn{2}{|c|}{ Descritivas } & \multicolumn{8}{|c|}{ Matriz de Correlações } & \multirow[t]{2}{*}{$\begin{array}{c}\text { Matriz } \\
\text { Fatoria }\end{array}$} \\
\hline & Media & DP & 1 & 2 & 3 & 4 & 5 & 6 & 7 & 8 & \\
\hline 1 Matrix span & 60.07 & 15.42 & & .653 & .715 & .589 & .617 & .610 & .632 & .548 & .748 \\
\hline 2 Letter span & 4.17 & 1.13 & .04 & & .826 & .542 & .680 & .707 & .753 & 679 & .838 \\
\hline 3 Digit span & 5.54 & 1.44 & .08 & .09 & & .527 & .709 & .734 & .726 & 695 & .872 \\
\hline 4 Matrix scan & 94.67 & 5.48 & .07 & .02 & .04 & & .611 & .597 & .559 & .600 & .674 \\
\hline $5 \mathrm{ABC}$ numerical & 67.94 & 24.09 & .03 & .04 & .03 & .01 & & .786 & .789 & .795 & .864 \\
\hline $6 \mathrm{ABCD}$ Gram & 77.7 & 21.36 & .04 & .03 & .02 & .006 & .02 & & .795 & .798 & .884 \\
\hline 7 Alphabet & 61.93 & 27.95 & .02 & .01 & .02 & .03 & .02 & .01 & & .774 & .876 \\
\hline 8 Digit ordering & 23.60 & 6.59 & .08 & .04 & .03 & .02 & .05 & .05 & .02 & & .856 \\
\hline PMA-R & 21.82 & 3.64 & & & & & & & & & \\
\hline Raven & 37.36 & 9.45 & & & & & & & & & \\
\hline
\end{tabular}

\section{Qual é o melhor preditor de $g$ (MCP ou MT) e por quê?}

Para responder a esta questão fizemos um outro estudo transcultural aumentando o número de tarefas de memória como também o número de provas cognitivas (Colom, FloresMendoza, Quiroga \& Privado, 2005). Assim, examinamos o desempenho de duas amostras independentes, a primeira composta por 76 participantes brasileiros (média de 23 anos; $D P=5,5$ ) e a segunda amostra composta por 63 participantes espanhóis (média de 20,9 anos; $D P=1,39$ ), em 12 tarefas de memória (seis MCP e seis MT) e em testes psicométricos de inteligência.

No caso da primeira amostra aplicaram-se os subtestes Séries, Cálculo, Vocabulário, Aritmética e Espacial da bateria BTAG (CEPA, 2001), e o Teste Equicultural de Inteligência (Cattell \& Cattell, 2002). No caso da segunda amostra aplicaram-se os subtestes Raciocínio Verbal, Relações Espaciais e Habilidade Numérica do DAT (Bennett, Seashore \& Wesman, 2000); os subtestes de Vocabulário, Rotação Mental, Computação e Raciocínio Indutivo do PMA (Thurstone, 1938 -versão informatizada por Quiroga, Privado, Beato, Colom \& Shih, 2004) e o teste de raciocínio quantitativo Moedas (Seisdedos,1980).

Transformamos os resultados de todas as tarefas em escores-z para estimar compostos de MCP, MT e $g$. Desta forma, obtivemos, para ambas as amostras, um escore de MCP (extraído de seis medidas) e um escore de MT (também extraído de seis medidas). O composto de $g$ para a primeira amostra foi extraído de seis medidas enquanto que para a segunda amostra o composto de $g$ foi extraído de 10 medidas.

Nossas análises mostraram que a correlação bruta obtida entre MCP e MT foi de 0,75 e de 0,68 para a primeira e segunda amostras, respectivamente. Portanto, ambos construtos compartilhavam fortemente as fontes de variância. A análise de regressão apontou que MT predizia $g$ um pouco melhor que MCP. No entanto, quando a correlação entre MCP e MT era estatisticamente controlada (no que se refere ao seu componente de armazenamento temporário), a força preditiva de MT era pequena. Este resultado corroborava uma suspeita acadêmica: o componente de armazenamento parece ser um fator crucial subjacente à relação entre MT e $g$.

Uma outra forma de investigação foi delineada (Colom, Abad, Rebollo, \& Shih, 2005). Desta vez, separamos os testes cognitivos por tipo de habilidades cognitivas. Assim, empregamos testes que avaliassem a Gc (teste de vocabulário do Primary Mental Ability e o teste de raciocínio verbal do Differential Abilities Battery), a Gf (teste de raciocínio do Primary Mental Abilities Battery e os subtestes 1 e 3 do Culture Fair Intelligence Test), a Gv (teste de rotação mental do PMA e teste de superfície) e a Gq ou inteligência quantitativa (teste de computação e teste Moedas).

O estudo contou com a participação de 208 estudantes universitários (idade média de 20,73; $D P=3,7$ ). A eles aplicamos, além das provas cognitivas, 12 medidas de memória (seis MCP e seis MT). Os dados foram submetidos a técnicas de CFA e de SEM. Assim, extraímos um fator latente de ordem superior para MCP e para MT $\left[\chi^{2}(46)=87,28\right.$; $\mathrm{CMIN} / d f=1,89$; RMSEA $=0,066]$. A correlação entre estes fatores de segunda ordem foi de 0,89 . No caso das habilidades cognitivas, verificamos que somente Gv e Gf foram os fatores de primeira ordem melhor preditos por $g\left[\chi^{2}(16)=33,48\right.$; $\mathrm{CMIN} / d f=2,09 ; \mathrm{RMSEA}=0,073]$.

No seguinte estágio de análise, estabelecemos os modelos estruturais para os fatores de inteligência e para os fatores de memória (MCP e MT) de ordem superior. O coeficiente estrutural de MCP para $g$ foi de $0,58\left[\chi^{2}(68)=100,49\right.$; $\mathrm{CMIN} / d f=1,48$; RMSEA $=0,048]$. Isso significava que o componente de armazenamento temporário capturado pelo fator latente de MCP comportou-se como um bom preditor de $g$. No caso de MT o coeficiente estrutural para $g$ foi de $0,89\left[\chi^{2}(69)=126,51 ; \mathrm{CMIN} / d f=1,83 ; \mathrm{RMSEA}=0,063\right]$. Tal resultado indicava que o composto de armazenamento + processamento de informação do fator latente de MT constituía o melhor preditor de $g$.

Considerando que a MCP e a MT compartilham o componente de armazenamento, procedeu-se a um modelo estrutural hierárquico em que MCP e MT prediziam $g$. Para 
tanto, todas as tarefas ingressaram como indicadores de MCP, mas unicamente as medidas de MT ingressaram como indicadores de MT.

Este modelo permite separar o componente de armazenamento das tarefas de memória de outros componentes da MT, de forma a verificar a capacidade preditiva tanto dos componentes de armazenamento quanto dos componentes de processamento do construto de MT. O modelo obtido mostrou-se adequado (Figura 2): $\left[\chi^{2}(154)=260,59\right.$; CMIN/ $d f=1,69 ; \mathrm{RMSEA}=0,058]$. Portanto, a MCP assim como a MT realmente predizem $g$. O coeficiente estrutural para MCP foi

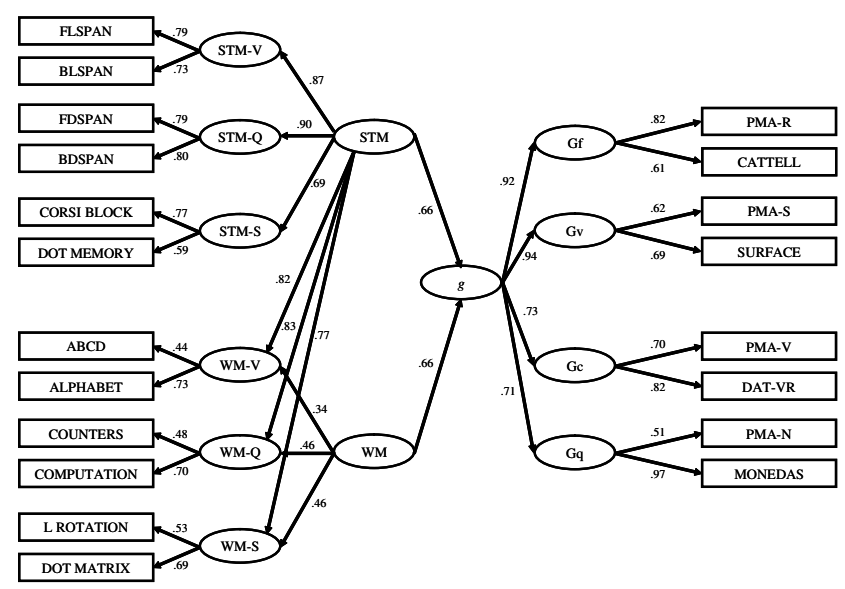

Figura 2. Modelo hierárquico para MCP e MT.

de 0,58 enquanto que o coeficiente estrutural para MT foi de 0,79 , embora ambos foram fixados como iguais $(0,66) \mathrm{sem}$, no entanto, perder a bondade de ajuste (Figura 2).

Assim sendo, os resultados mostraram que quando na análise estrutural a MCP ingressa com todas as medidas de capacidade de memória, o valor do coeficiente não se altera. Permanece o mesmo valor que se obteve quando a MCP ingressou somente com as medidas de armazenamento $(0,58$ ou 0,66$)$. No caso da MT, o coeficiente muda na análise estrutural. O valor passa de 0,89 para 0,66 . Mais ainda, a relação entre MT e $g$ é consistente com o isomorfismo entre ambos construtos quando se inclui a combinação armazenamento + processamento. Contudo, esse isomorfismo desaparece quando se controla o componente de armazenamento, passando de 0,89 a 0,66 .

Finalmente, esse estudo revelou que o componente específico de MT também prediz $g$, embora em menor intensidade, $\mathrm{o}$ que impele necessariamente a fazer o seguinte questionamento: Qual é a natureza desse componente adicional? O grupo de Engle é partidário de que esse componente adicional seria o controle atencional, enquanto que autores como Ackerman e cols. (2002) e nossa equipe (Colom, Rebollo, Palacios, JuanEspinosa \& Kyllonen, 2004) propõem que é a velocidade.

Levando em consideração, portanto, os resultados obtidos no estudo empírico que acabamos de comentar, se delineou um novo estudo empírico destinado a identificar se o armazenamento e a velocidade eram necessários e suficientes para explicar a relação entre MT e inteligência. Se a resposta fosse positiva, então o grupo de Engle e cols. (1999) poderia estar equivocado uma vez que não haveria necessidade de recorrer ao conceito de controle atencional.

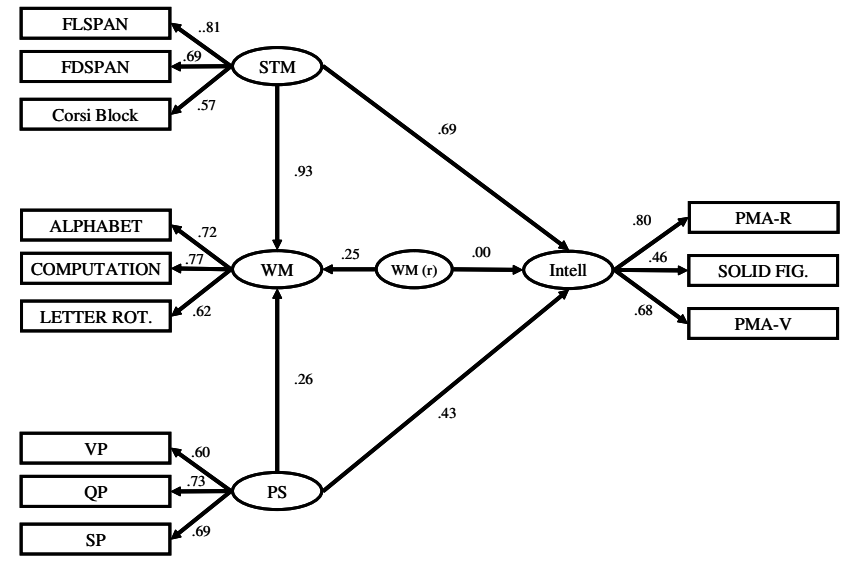

Figura 3. Modelo de quatro fatores para MCP, MT, PS e $g$.

Nota da Figura 2. FLSPAN = forward letter span, BLSPAN = backward letter span, FDSPAN = forward digit span, $\mathrm{BDSPAN}=$ backward digit span, CORSI BLOCK = tarefa de memorização de seqüências de posições, DOT MEMORY = tarefa de memorização da situação de uma série de pontos, $\mathrm{ABCD}=$ tarefa de $\mathrm{ABCD}, \mathrm{ALPHABET}=$ tarefa de alfabeto, COUNTERS $=$ tarefa de contadores, COMPUTATION $=$ tarefa de amplitude de cálculo, $\mathrm{L}$ ROTATION = tarefa de rotação de letras e memorização de posições, DOT MATRIX = tarefa de verificação de matrizes e memorização de pontos, STM-V = memória de curto prazo verbal, $\mathrm{STM}-\mathrm{Q}=$ memória de curto prazo numérica, STM-S = memória de curto prazo espacial, WM-V = memória de trabalho verbal, $\mathrm{WM}-\mathrm{Q}=$ memória de trabalho numérica, $\mathrm{WM}-\mathrm{S}=$ memória de trabalho espacial, STM = memória de curto prazo, WM = memória de trabalho, $g=$ fator geral de inteligência, $\mathrm{Gf}$ = inteligência fluida, $\mathrm{Gv}$ = inteligência espacial, $\mathrm{Gc}=$ inteligência cristalizada, $\mathrm{Gq}=$ inteligência numérica, $\mathrm{PMA}-\mathrm{R}=$ subteste de raciocínio indutivo do Primary Mental Abilities Battery, CATTELL = subtestes 1 e 3 do teste livre de influências culturais de R.B. Cattell, PMA-S = subteste de rotação mental do Primary Mental Abilities Battery, SURFACE $=$ teste de desenvolvimento de superfícies, $\mathrm{PMA}-\mathrm{V}=$ subteste de vocabulário do Primary Mental Abilities Battery, DAT-VR = subteste de raciocínio verbal da Differential Aptitude Test Battery, PMA-N = subteste de cálculo do Primary Mental Abilities Battery, MONEDAS = subteste de raciocínio numérico.

Todos os coeficientes de regressão estão estandarizados. Os valores residuais foram omitidos visando uma maior clareza dos resultados. E eles podem ser facilmente calculados: eleva-se ao quadrado os valores dos coeficientes de regressão que alcançam uma determinada variável endógena ou exógena. No caso de haver mais de um valor, deve-se somar o resultado e subtrai-lo de 1. Por exemplo: $0,66^{2}+0,66^{2}=0,86, \log 01-0,86=0,14$ constituiria o residual de $g$.

Nota da Figura 3. FLSPAN $=$ forward letter span, FDSPAN $=$ forward digit span, CORSI BLOCK = tarefa de memorização de seqüências de posições, ALPHABET $=$ tarefa de alfabeto, COMPUTATION $=$ tarefa de amplitude de cálculo, LETTER ROT = tarefa de rotação de letras e memorização de posições, $\mathrm{VP}=$ velocidade de reconhecimento verbal, $\mathrm{QP}=$ velocidade de reconhecimento numérico, $\mathrm{SP}=$ velocidade de reconhecimento espacial, $\mathrm{STM}=$ memória de curto prazo, $\mathrm{WM}=$ memória de trabalho, $\mathrm{PS}=$ velocidade de reconhecimento, WM (r) = residual do fator latente da memória de trabalho, Intell $=$ inteligência, PMA-R = subteste de raciocínio indutivo do Primary Mental Abilities Battery, SOLID FIG = rotação de figuras maciças, PMA-V = subteste de vocabulário do Primary Mental Abilities Battery.

Todos os coeficientes de regressão estão padronizados. Os valores residuais são omitidos visando uma maior clareza dos resultados, uma vez que eles podem facilmente ser calculados seguindo a estratégia comentada nas figuras anteriores.

Na nossa nova hipótese, a MCP junto com a PS seriam necessárias e suficientes para explicar a relação entre MT e inteligência. Assim, estudamos o desempenho de 111 participantes (idade média de 18,$00 ; D P=2,7$ ) em três tarefas de MCP, três de MT e em três tarefas de PS (Colom, Abad, Shih, 2006). A inteligência também foi representada median- 
te três tarefas de Gf, Gv e Gc. A precisão das tarefas, após normalização das tarefas de velocidade de processamento, girou em torno de 0,73 a 0,91 . Esses dados foram, então, submetidos a análise SEM.

Primeiramente explorou-se a relação entre MCP, MT e PS ao nível da variável latente. $O$ modelo mostrou-se adequado: $\left[\chi^{2}(24)=27,77 ; \mathrm{CMIN} / d f=1,16 ;\right.$ RMSEA $\left.=0,04\right]$. Encontrouse uma forte relação entre MCP e MT $(0,89)$ e uma baixa associação, porém significativa, entre MT e PS $(-0,31 ; p<$ $0,05)$; enquanto que a relação entre MCP e PS não foi significativa $(-0,12 ; p>0,05)$. O coeficiente estrutural entre $g$ e MCP foi de 0,65; com MT foi de 0,82 e com PS foi de -0,42. Portanto, MCP, MT e PS estão relacionados a $g$.

Novamente, considerando que MT compreende os componentes de armazenamento e de processamento transitório de informação foi necessário controlá-los por meio do seguinte modelo: MCP e PS predizem MT e a variância restante não-predita pelos fatores latentes deixaria um residual que representaria a variância única de MT. O objetivo era verificar se ainda existia uma variância compartilhada entre o MT-residual e o $g$. O modelo provou-se adequado: $\left[\chi^{2}(50)=76,06\right.$; $\mathrm{CMIN} / d f=1,52$; RMSEA $=0,069]$. O coeficiente estrutural entre MCP e $g$ foi de 0,69 (um valor consistente com os observados previamente por nossa equipe) e o coeficiente estrutural entre PS e $g$ foi de $-0,43$. Mais ainda, o coeficiente estrutural entre o resíduo de MT e $g$ pôde ser fixado em 0.

Estes resultados indicaram, portanto, que MCP e PS predizem $g$, enquanto que o fator latente de MT (com seus componentes de armazenamento e processamento controlados) não apresenta predição significativa (Figura 3). Em outras palavras, para que MT e $g$ apresentem alta associação se faz necessária a participação da capacidade de armazenamento (característica de MCP) e da velocidade de processamento (PS). Dessa forma, não ocorre variância restante para explicar, o que torna desnecessário recorrer a conceitos como o controle de atenção.

Considerando que os estudos registrados na literatura apresentam diversidade de metodologia de análise que impede uma conclusão mais acurada dos resultados, decidimos re-analisar as matrizes dos estudos mais importantes publicados sobre MT e $g$ (Colom, Rebollo, Abad e Shih, no prelo). A seleção desses estudos esteve baseada em dois critérios: 1) os estudos deviam considerar tarefas de memória simples (MCP) e complexas (MT) e, 2) os estudos deviam considerar medidas de habilidades cognitivas. A partir desses critérios, foram selecionadas as matrizes dos seguintes estudos: Engle e cols. (1999), Miyake e cols. (2001), Conway e cols. (2002), Bayliss, Jarrold, Gunn \& Baddeley (2003) e Kane e cols. (2004). Os dados foram submetidos a Análise Hierárquica-Schmid Leiman.

A Tabela 3 mostra que MCP e MT não são claramente distinguíveis. Ambas as medidas dividem alguma coisa em comum que permite sua associação com medidas de habilidades cognitivas. $\mathrm{Na}$ análise das cinco matrizes, observa-se que MCP e MT carregam em igual magnitude no fator de segunda ordem.

Por outro lado, também se contrastaram uma serie de SEM, usando como referência o modelo empregado por Conway e cols. (2002), assim como por Colom, Abad, Rebollo e Shih (2005). Neste modelo-base, o fator latente de MCP está definido por todas as tarefas de memória, enquanto que o fator latente de MT fica definido unicamente pelas tarefas de MT. Os resultados foram unânimes ao assinalar que as diferenças individuais em uma série de capacidades cognitivas são preditas de maneira consistente por MCP, mas

Tabela 3. Análise Hierárquica-Schmid Leiman das cinco matrizes de dados.

\begin{tabular}{|c|c|c|c|c|c|}
\hline \multirow{2}{*}{ Matrizes } & \multirow{2}{*}{ Tarefas utilizadas } & \multirow{2}{*}{$\begin{array}{l}\text { Variância explicada pelo } \\
\text { fator de segunda ordem }\end{array}$} & \multicolumn{3}{|c|}{ Peso no fator de segunda ordem } \\
\hline & & & MCP & MT & Hab. Cog. \\
\hline $\begin{array}{l}\text { Engle e cols. } \\
\text { (1999) }\end{array}$ & $\begin{array}{l}16 \text { medidas: } \\
\text { Tarefas MT e MCP } \\
\text { Cattell's Culture Fair Test } \\
\text { Progressive Matrices (SPM) }\end{array}$ & $26,81 \%$ & 0,573 & 0,532 & 0,537 \\
\hline $\begin{array}{l}\text { Miyake e cols. } \\
(2001)\end{array}$ & $\begin{array}{l}12 \text { medidas: } \\
\text { Tarefas MT e MCP } \\
\text { Torres de Hanói } \\
\text { Testes de visualização espacial } \\
\text { Testes de relações espaciais } \\
\text { Testes de velocidade perceptual }\end{array}$ & $27,83 \%$ & 0,481 & 0,507 & 0,575 \\
\hline $\begin{array}{l}\text { Conway e cols. } \\
\text { (2002) }\end{array}$ & $\begin{array}{l}13 \text { medidas: } \\
\text { Tarefas MT, MCP e PS } \\
\text { Cattell's Culture Fair Test } \\
\text { Progressive Matrices (SPM) }\end{array}$ & $19,15 \%$ & 0,528 & 0,560 & 0,302 \\
\hline $\begin{array}{l}\text { Bayliss e cols. } \\
(2003)\end{array}$ & $\begin{array}{l}11 \text { medidas: } \\
\text { Tarefas MT, MCP, PS } \\
\text { Progressive Matrices (CPM) } \\
\text { Reading and Math Test }\end{array}$ & $31,90 \%$ & 0,510 & 0,523 & 0,644 \\
\hline $\begin{array}{l}\text { Kane e cols. } \\
\text { (2004) }\end{array}$ & $\begin{array}{l}25 \text { medidas: } \\
\text { Tarefas MT, MCP } \\
\text { Progressive Matrice (SPM) } \\
\text { WAIS } \\
\text { Testes de inteligência cristalizada } \\
\text { Testes de habilidade espacial }\end{array}$ & $33,60 \%$ & 0,612 & 0,634 & 0,533 \\
\hline
\end{tabular}


não pelo fator de MT (aquele independente do armazenamento transitório). A enorme vantagem destas re-análises é que as distintas bases de dados analisadas foram exploradas a partir do mesmo modelo SEM de referência, superando desse modo a tendência a que os modelos de referência mudem de estudo para estudo (Cowan, 2005). Portanto, o resultado desta re-analise é consistente com a nossa visão teórica de que o componente de armazenamento da MT resulta crucial para compreender a alta relação entre MT e $g$.

\section{Conclusão}

Os diversos esforços investigativos enveredados até o momento por nossa equipe, mostram existir uma capacidade geral que explica uma grande quantidade de diferenças individuais observadas em tarefas de capacidade de memória e em provas de habilidades cognitivas. Além disso, MT prediz $g$ com tamanha força que ambos construtos podem ser considerados isomórficos. Contudo esse isomorfismo decai quando o componente de armazenamento de MT é controlado, dando passagem para perspectiva de que a relação entre MT e $g$ depende de alguns componentes. Nossos estudos revelam que esses componentes seriam a capacidade de armazenamento e a velocidade de processamento.

Estas observações, empiricamente testadas, contradizem os resultados de estudos como o de Kane e cols (2004) que afirma ser a variância compartilhada entre MCP e MT um reflexo do funcionamento executivo e não de mecanismos de armazenamento. Nossos estudos revelam que a relação entre MT e $g$, ao nível da variável latente, é quase a mesma que se encontra em modelos SEM de três e quatro fatores. O mesmo ocorre com a PS no que se refere a modelo SEM de quatro fatores.

Nossos resultados tampouco validam a assunção de Engle e cols. (1999) que afirmam que MCP pouco explica a relação entre MT e inteligência. Nossas análises mostram que o componente residual de MT, após o controle do componente de armazenamento e de velocidade de processamento, relaciona-se, de maneira não significativa, a $g$. Nessa situação a MCP prediz $g$ melhor que MT (com seu componente de armazenamento controlado). Considerando as diversas análises das provas de MT, MCP, PS e $g$, podemos corroborar o que afirmamos em 2001: os componentes de armazenamento de curto prazo e a velocidade de processamento são necessários e suficientes para explicar a relação entre MT e $g$.

\section{Referências}

Ackerman, P. L.; Beier, M. E. \& Boyle, M. O. (2002). Individual differences in working memory within a nomological network of cognitive and perceptual speed abilities. Journal of Experimental Psychology: General, 131(4), 567-589.

Ackerman, P. L.; Beier, M. E. \& Boyle, M. O. (2005). Working memory and intelligence: The same or different constructs? Psychological Bulletin, 131(1), 30-60.

Anderson, J. R. (1990). Cognitive psychology and its implications. New York: Freeman.

Bayliss, D. M.; Jarrold, C.; Gunn, D. \& Baddeley, A. (2003). The complexities of complex span: Explaining individual differences in working memory in children and adults. Journal of Experimental Psychology: General, 132(1),71-92.

Bennett, G. K.; Seashore, H. G. \& Werman, A. G. (2000). Test de aptitudes mentales diferenciales. Madrid: TEA.

Browne, M. W.; \& Cudeck, R. (1992). Alternative ways of assessing model fit. Sociological Methods and Research, 21, 230-258.

Carpenter, P. A.; Just, M. A. \& Shell, P. (1990). What one intelligence test measures: a theorical account of the processing in the Raven Progressive Matrices Test. Psychological Review, 97(3), 404-431.

Carroll, J. B. (1993). Human Cognitive Abilities. Cambridge: Cambridge University Press.

Cattell, R. B. \& Cattell, A. K. S. (2002). Teste Equicultural de Inteligência. Adaptado pelo Centro de Psicologia Aplicada [Culture Fair Intelligence Test]. RJ: CEPA.

Centro Editor de Psicologia Aplicada (2002). Bateria de Testes de Aptidão Geral-BTAG II (GATB Battery).Adaptado por Gustavo Lisboa Braga [General Aptitude Test Battery]. RJ: CEPA.

Colom, E.; Flores-Mendoza, C. E.; Quiroga, M. A. \& Privado, J. (2005). Working memory and general intelligence: The role of short-term storage. Personality and Individual Differences, 39(5), 1005-1014.

Colom, R. \& Shih, P. C. (2004). Is working memory fractioned onto different components of intelligence? A reply to Mackintosh and Bennett. Intelligence, 31, 431-444.

Colom, R.; Abad, F.J. \& Shih, P.C. (2006, janeiro). Short-term memory and processing speed predict the relationship between working memory and intelligence. Trabalho apresentado na VIII Jornada de la Sociedad Española para la Investigación de las Diferencias Individuales. Madrid, Espanha.

Colom, R.; Flores-Mendoza, C. E. \& Rebollo, I. (2003). Working memory and intelligence. Personality and Individual Differences, 34, 33-39.

Colom, R.; Rebollo, I.; Abad, F. J. \& Shih, P. C. (no prelo). Complex span tasks, simple span task, and cognitive abilities: A reanalysis of key studies. Memory \& Cognition.

Colom, R.; Rebollo, I.; Palacios, A.; Juan-Espinosa, M. \& Kyllonen, P. C. (2004). Working memory is (almost) perfectly predicted by g. Intelligence, 32, 277-296.

Colom, R.; Abad, F. J.; Rebollo, I. \& Shih, P. C. (2005). Memory span and general intelligence: A latent-variable approach. Intelligence, 33, 623-642.

Colom, R. \& Flores-Mendoza, C. (2001). Inteligencia y memoria de trabajo: la relación entre factor $\mathrm{G}$, complejidad cognitiva y capacidad de procesamiento. Psicologia: Teoria \& Pesquisa, 17(1), 37-47.

Colom, R. \& Flores-Mendoza, C. (2006). Processamento humano de informação e inteligência. Em C. Flores-Mendoza \& R. Colom (Orgs.), Introdução à Psicologia das Diferenças Individuais (pp. 101-130). Porto Alegre: ArtMed.

Colom, R.; Shih, P. C.; Flores-Mendoza, C. E. \& Quiroga, M. A. (no prelo). The real relationship between short-term memory and working memory. Memory.

Conway, A. R. A.; Cowan, N.; Bunting, M. F.; Therriault, D. J. \& Minkoff, S. R. B. (2002). A latent variable analysis of working memory capacity, short-term memory capacity, processing speed, and general fluid intelligence. Intelligence, 30(2),163183.

Cowan, N. (1995). Attention and memory: An integrated framework. Oxford: Oxford University Press. 
Cowan, N. (2005). Working memory capacity. Hove, East Sussex: Psychology Press.

Engle, R. W.; Tuholski, S. W.; Laughlin, J. E. \& Conway, A. R. A. (1999). Working memory, short-term memory, and general fluid intelligence: A latent-variable approach. Journal of Experimental Psychology: General, 128(3), 309-331.

Jensen, A. \& Weng, L. (1994). What is a good $g$ ?. Intelligence, 18, 231-258.

Kane, M. J.; Hambrick, D. Z.; Tuholski, S. W.; Wilhelm, O.; Payne, T. W. \& Engle, R. W. (2004). The generality of working memory capacity: A latent-variable approach to verbal and visuospatial memory span and reasoning. Journal of Experimental Psychology: General, 133, 189-217.

Kyllonen, P. C. \& Christal, R. (1990). Reasoning ability is (little more than) working memory capacity?!. Intelligence, $14,89-$ 433.

Kyllonen, P. C. (1996) Is working memory capacity Spearman'g? Em I. Dennis \& P. Tapsfield (Orgs.), Human abilities: their nature and measurement (pp. 49-75). Mahwah: Erlbaum.

Mackintosh, N. J. \& Bennett, E. S. (2003). The fractionation of working memory maps onto different components of intelligence. Intelligence, 31, 519,531.

Miyake, A.; Friedman, N. P.; Rettinger, D. A.; Shah, P. \& Hegarty, M. (2001). How are visuospatial working memory, executive functioning, and spatial abilities related? A latent-variable analysis. Journal of Experimental Psychology: General, 130(4), 621-640

Mukunda, K.V. \& Hall, V. C. (1992). Does performance on memory for order correlate with performance on standardized measures of ability? A meta-analysis. Intelligence, 16, 81-97.
Quiroga, M. A.; Privado, J.; Beato, M. S.; Colom, R. \& Shih, P. C. (2004). Test de aptitudes mentales primarias, Versión informática. Madrid: TEA.

Richardson, J. T. E. (1996). Evolving Issues in Working Memory. Em J. T. E. Richardson \& cols. (Orgs.), Working Memory and Human Cognition (pp. 120-154). New York: Oxford University Press.

Seamon, J. G. \& Kenrick, D. T. (1994). Psychology. Englewood Cliffs: Prentice Hall.

Seisdedos, N. (1980). Monedas. Madrid: TEA.

Shah, P. \& Miyake, A. (1996). The separability of working memory resources for spatial thinking and language processing: An individual differences approach. Journal of Experimental Psychology: General, 125, 4-27.

Stauffer, J.; Ree, M. \& Carretta, T. (1996). Cognitive-components tests are not much more than $g$ : An extension of Kyllonen's analyses. The Journal of General Psychology, 123(3), 193205.

Süß, H.; Oberauer, K.; Wittman, W.; Wilhelm, O. \& Shulze, R. (2002). Working memory capacity explains reasoning ability -and a little bit more. Intelligence, 30, 261-288.

Thurstone, L. (1938). Primary mental abilities. Psychometrics Monographs 1. Madrid: TEA.

Recebido em 15.03.2005

Primeira decisão editorial em 10.01.2006

Versão final em 29.03.2006

Aceito em 04.04.2006

\section{CONGRESSO LATINO-AMERICANO DE PSICOLOGIA JUNGUIANA}

Data: 3 a 7 de setembro de 2006

Local: Hotel Conrad Resort \& Casino de Punta del Este Uruguay

\section{Organização}

Sociedade Uruguaia de Psicologia Analítica (SUPA)

Informações e inscrições

www.congresos-rohr.com/psicoljunguiana

information@congresos-rohr.com 


\section{Anexo 1 - Exemplos de tarefas MCP, MT e PS}

\section{MCP-Verbal (Letter Span)}

Várias letras são seqüencialmente mostradas na tela do computador. A tarefa começa com três letras, incrementando-se o número de letras até que o participante não possa reproduzir a seqüência no teclado (três erros de cinco tentativas).

\section{MCP-Numérica (Digit span)}

Vários dígitos são seqüencialmente mostrados na tela do computador. A tarefa começa com três dígitos, incrementado-se a quantidade de números até que o participante não possa reproduzir a sequiência no teclado (três erros de cinco tentativas).

\section{MCP-Viso-espacial (Matrix span)}

Cinco quadrados vazios são mostrados no meio da tela do computador em posição horizontal e um outro vazio na parte superior da tela. Depois de um sinal de aviso, os cinco quadrados vazios são seqüencialmente preenchidos por uma matriz de $4 \times 4$ células (cada quadrado por 1s). Na medida que um quadrado é preenchido o anterior desaparece. $\mathrm{O}$ intervalo inter-estímulo é de 500ms. Para evitar aprendizagem, a seqüência dos estímulos (matrizes preenchidas) nos quadrados é apresentada aleatoriamente considerando 15 possíveis matrizes. Após a apresentação dos cinco estímulos, o quadrado que está na parte superior da tela mostrará um desses estímulos para o qual o participante indicará sua posição dentre os cinco quadrados.

\section{MT-Verbal (ABCD)}

Duas categorias e cinco palavras são utilizadas. As categorias são "árvore" e "comida". Logo se apresenta uma frase com uma das categorias: "O cedro precede ao mogno" (AB). Depois a seguinte categoria: "O alho não precede ao sal" (DC). Por último, se apresenta a ordem das categorias: "As árvores não precedem à comida" (DCAB). Apresentam-se oito alternativas. $\mathrm{O}$ sujeito deverá indicar a combinação correta dos pares $\mathrm{AB}$ e $\mathrm{CD}$, assim como a sua posição.

\section{MT-Numérica (Computation)}

A tarefa inclui verificação e recordação. Verifica-se, durante seis segundos, se a equação mostrada é correta e recorda-se a solução mostrada, mesmo não sendo correta. Terminada a última equação, solicita-se do participante que indique a seqüência serial das soluções mostradas de todas as equações. Cada equação inclui duas operações e utiliza dígitos de 1 a 10. A solução sempre é número de um dígito.

\section{MT-Visuo-espacial (Letter rotation)}

Apresentam-se letras maiúsculas que podem estar na forma normal ou em espelho e podem estar girando em ângulos de $0^{\circ}, 45^{\circ}, 90^{\circ}$ ou $180^{\circ}$. A tarefa é verificar a forma (normal ou em espelho) e a posição (distância angular).

\section{PS}

Apresentam-se seqüencialmente diversas letras, cada uma por 650ms. Logo, se apresenta um delay de 500ms. Aparece uma letra e pergunta-se ao participante se essa letra pertence à série de letras anteriormente apresentada. As séries eram compostas de três a cinco letras. O escore é a média do tempo de reação para respostas corretas. 